\title{
Spin doping of individual molecules by using single-atom manipulation
}

\author{
Roberto Robles, ${ }^{* \dagger}$ Nicolás Lorente, ${ }^{\dagger}$ Hironari Isshiki, ${ }^{\ddagger}$, Jie Liu, ${ }^{\ddagger}, \uparrow$ Keiichi \\ Katoh," Brian K. Breedlove, Masahiro Yamashita, " and Tadahiro Komeda ${ }^{\ddagger}$, \\ $†$ Centro de Investigación en Nanociencia y Nanotecnología, CIN2 (CSIC - ICN), Campus \\ de la UAB, E-08193 Bellaterra, Spain \\ $\ddagger$ Institute of Multidisciplinary Research for Advanced Materials (IMRAM, Tagen), Tohoku \\ University, 2-1-1, Katahira, Aoba-Ku, Sendai 980-0877, Japan \\ I Department of Chemistry, Graduate School of Science, Tohoku University, \\ Aramaki-Aza-Aoba, Aoba-Ku, Sendai 980-8578, Japan \\ $\S$ CREST, Japan Science and Technology Agency (JST), Saitama 332-0012, Japan \\ E-mail: roberto.robles@cin2.es
}

\begin{abstract}
Being able to control the spin of magnetic molecules at the single-molecule level will make it possible to develop new spin-based nanotechnologies. Gate-field effects and electron and photon excitations have been used to achieve spin switching in molecules. Here, we show that atomic doping of molecules can be used to change the molecular spin. Furthermore, a scanning tunneling microscope was used to place or remove the atomic dopant on the molecule, allowing us to change the molecular spin in a controlled way. Bis(phthalocyaninato)yttrium ( $\left.\mathrm{YPc}_{2}\right)$ molecules deposited on an $\mathrm{Au}(111)$ surface keep their spin-1/2 magnetic moment due to the small molecule-substrate interaction. However, when Cs atoms were carefully placed onto $\mathrm{YPc}_{2}$ molecules, the spin of the
\end{abstract}


molecule vanished as shown by our conductance measurements and corroborated by the results of density functional theory calculations.

\section{Keywords}

spin doping,atomic manipulation,metal-organic molecules,Kondo,STM

In molecular devices that use molecular magnetic moments, such as spin valves, ${ }^{1-3}$ the electron current is controlled via the intrinsic spin. Hence, active control of the molecular spin is crucial for determining the functionalities of the molecular device. ${ }^{3}$ Recently, in breakjunction experiments, molecules have been placed in a transistor setup, in which a gate field is applied to modify the molecular properties. ${ }^{4}$ By setting the operational regime of their molecular device, the authors could control the molecular moment of the connected molecule and, hence, control the magnetism at the single-molecule level. Changing the conformation of a molecule is another efficient method for modifying its spin state. Controlled elongation of cobalt complexes can be used to alter the spin of these molecules. ${ }^{5}$ Moreover, it has been shown that molecular conformation is affected by photon ${ }^{6}$ and electron excitations. ${ }^{7}$ Indeed, photons can be used to change the conformation of an azobenzene derivative adsorbed on an $\mathrm{Au}(111)$ surface, ${ }^{6}$ and electronic excitation induce changes in molecules, such as double-decker bis(phthalocyaninato)terbium $\left(\mathrm{TbPc}_{2}\right)$ molecules absorbed on $\mathrm{Au}(111) .^{7}$ $\mathrm{TbPc}_{2}$ molecules have been excited by injecting an electron current large enough to rotate one of the Pc ligands, causing a shift in the orbital levels, which quenches the molecular spin. In other words, modifying the structure of a molecule affects its electronic levels, leading to different spin states. Hence, the ultimate goal is to directly access the electronic levels that carry the spin information. Electron doping is, thus, an excellent way to change the electronic and magnetic properties of a molecule.

Molecular thin films have been doped with molecules ${ }^{8-12}$ and alkali metals. ${ }^{13-17}$ Doping a monolayer of molecules is conceptually very different because the concentration of dopants 

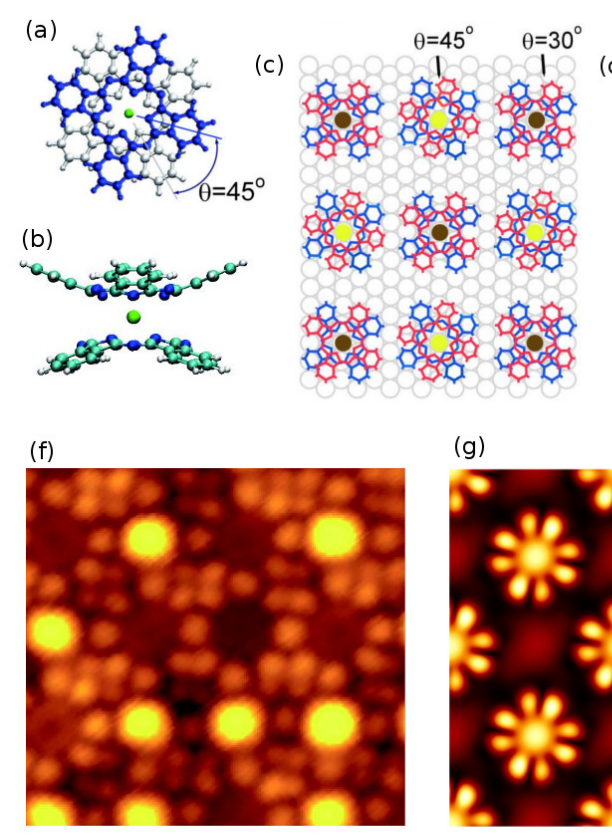

(g)

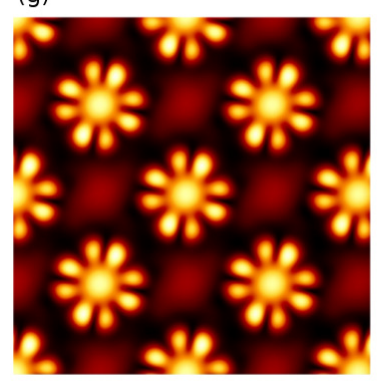

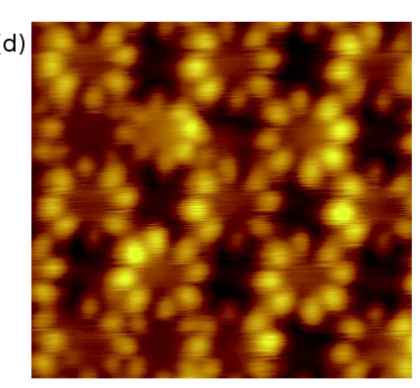

(e)

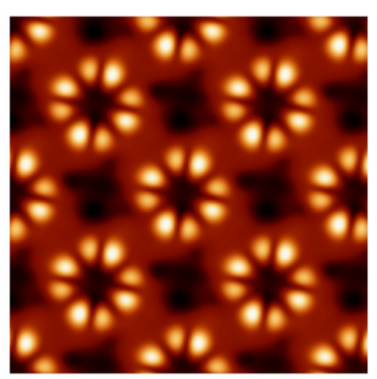

(h)
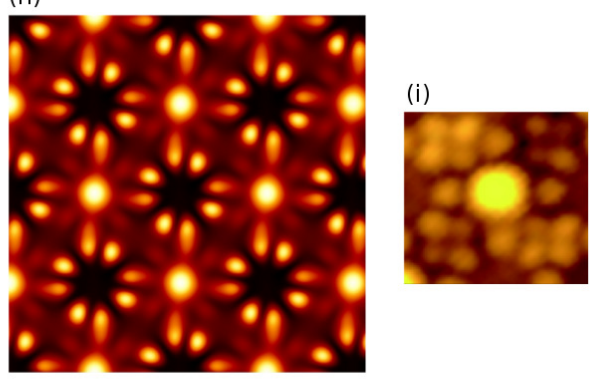

Figure 1: Drawing of a $\mathrm{YPc}_{2}$ molecule $(a)$ from above in the orientation of molecular adsorption, and (b) from the side. The two Pc ligands of the molecule form an angle, $\theta$, of $45^{\circ}$, and the Pc ligands bend to bind to coordinate to the Y ion. (c) On the Au (111) surface, the molecule arrange so that molecules with $\theta=45^{\circ}$ and $\theta=30^{\circ}$ alternate. Hence, the shown area contains four molecules with $\theta=45^{\circ}$, of which the centers are colored yellow. (d) In the STM image, the molecules with $\theta=45^{\circ}$ are bright, whereas the ones with $\theta=30^{\circ}$ are dark. (e) In an STM image simulated using DFT calculations, a molecule with $\theta=45^{\circ}$ is surrounded by ones with $\theta=30^{\circ}$. Only the molecules with $\theta=45^{\circ}$ are clearly visible in the constant current STM simulations, similar to $(d)$. $(f)$ STM image with Cs atoms adsorbed on the molecules (bright spots). The same pattern was reproduced by simulations. Simulated STM images with Cs atoms sitting on top of $(g) \theta=45^{\circ}$ and $(h) \theta=30^{\circ}$ molecules. When Cs atoms sit on $\theta=30^{\circ}$ molecules, they light up. Overlap of the protrusions of the molecules with $\theta=30^{\circ}$ and $\theta=45^{\circ}$ causes a cross-like pattern, which is easier to see when the Cs atoms sits on a $\theta=30^{\circ}$ molecule. ( $i$ ) Magnified experimental image of a Cs atom sitting on a $\mathrm{YPc}_{2}$ molecule. 
is the same as that of molecules. Moreover, with the development of scanning tunneling microscopy (STM), dopant effects at the single-molecule level can be fully characterized. Hence, whether the dopant is inducing a mere shift in the electronic levels with charge injection or whether the interactions are strong enough to cause the formation of another molecular species can be determined.

In the present work, we showed that $\mathrm{Cs}$ atoms adsorbed on $\mathrm{YPc}_{2}$ molecules on an $\mathrm{Au}$ (111) surface. A single electron per molecule was injected in the molecular $\pi$-system while the general molecular properties, such as the conformation and molecular chemistry, were maintained. In other words, although the $\mathrm{Cs}$ atom $/ \mathrm{YPc}_{2}$ molecule ratio is one, the Cs atoms act as dopants and donate an electron to a molecule, causing a shift in energy levels, which has been observed in the case of Cs doping of copper-Pc thin films. ${ }^{15,17}$ Thus, it should be possible to control the injection of charge in a single molecule without using complicated techniques, such as gate electrodes ${ }^{4}$ and conformational changes via external excitations. ${ }^{6,7}$

In contrast to $\mathrm{TbPc}_{2}, \mathrm{YPc}_{2}$ molecules are not single-molecule magnets but paramagnets because they do not contain $f$ electrons. However, they constitute an open-shell $\pi$-electronic system; there is an unpaired electron in a $\pi$ orbital of the Pc ligand, which is henceforth called the singly occupied molecular orbital ( $\mathrm{SOMO})$. $\mathrm{YPc}_{2}$ is relatively inert and adsorbs on $\mathrm{Au}$ (111) surfaces in a manner similar to that of the $\mathrm{TbPc}_{2}$ molecule. ${ }^{7,18}$ In the monolayers, the molecules were arranged with an alternating angle between the two Pc ligands, $\theta$, of $45^{\circ}$ and $30^{\circ}$, leading to a checkerboard pattern, ${ }^{7}$ as shown in Figure 1 . The $\theta=30^{\circ}$ molecules have a SOMO-LUMO gap, and the spin is quenched via electron transfer from the substrate. ${ }^{7}$ However, the $\theta=45^{\circ}$ molecules have an electron in the $\pi$ orbital of the Pc ligand. Scanning tunneling spectroscopy (STS) spectra for $\mathrm{YPc}_{2}$ molecules with $\theta=45^{\circ}$ showed a zero-bias anomaly, which was assigned to be a Kondo peak due to the unpaired electron, as shown in Figure 2. When Cs adsorbs onto a $\theta=45^{\circ}$ molecule, a single electron is transferred, quenching the spin. Moreover, the STM tip could be used to control the location of the Cs adatom and, hence, to switch on and off the molecular spin. 
All experiments were done at cryogenic temperatures $(4.7 \mathrm{~K})$ in an ultra-high vacuum (UHV). A gold single crystal was used for the substrate, and its (111) surface was prepared by $\mathrm{Ar}^{+}$sputtering and annealing. The synthesis of $\mathrm{YPc}_{2}$ is described elsewhere. ${ }^{19} \mathrm{YPc}_{2}$ molecules were deposited onto the Au (111) surface by evaporation from a Ta boat. The flux rate was kept at $\sim 0.1$ monolayers (MLs) per minute, and the Au substrate was kept at room temperature. No further annealing was performed after deposition. Topological STM images were obtained in the constant current mode. STS spectra were obtained using a lock-in amplifier technique with a modulation voltage of $1 \mathrm{mV}$. Cs was evaporated from a SAES Getter source (SAES Getters Group) on the $\mathrm{YPc}_{2}$ film at room temperature. The deposited amount was monitored by a quartz-crystal thickness monitor. More information can be found in the supporting online material.

Calculations were performed using density functional theory (DFT) as implemented in the VASP code. ${ }^{20}$ The PBE flavor of the generalized gradient approximation ${ }^{21}$ was used, and the forces were corrected for the missing van der Waals interactions using the Grimme scheme. ${ }^{22,23}$ The unit cell used for the simulations contained one molecule with $\theta=45^{\circ}$ and one with $\theta=30^{\circ}$. Please refer to the supporting online material for more details of the calculations.

Figures $1(a)$ and $(b)$ show drawings of the molecule with $\theta=45^{\circ}$, in which two Pc ligands sandwich a $\mathrm{Y}^{3+}$ ion. The monolayer is depicted in Figure $1(c)$, and the color of the dot in the center of a molecule indicates whether the molecule is bright (yellow) or dark (brown) in the constant current STM image taken at $-0.8 \mathrm{~V}$ (Figure $1(d)$ ). Agreement between simulated ${ }^{24}$ and experimental STM images shows that the computed geometrical and electronic structures are consistent with the experimental ones. Thus, we were able to see the difference in molecular contrast between molecules with $\theta=45^{\circ}$ (bright) and $\theta=30^{\circ}$ (dark), which is due to the steeper angle of the upper Pc ligand in the latter than that in the former, making it taller. The image is dominated by contributions of the highest occupied molecular orbital (HOMO), which gives more contrast than higher lying orbitals because it 


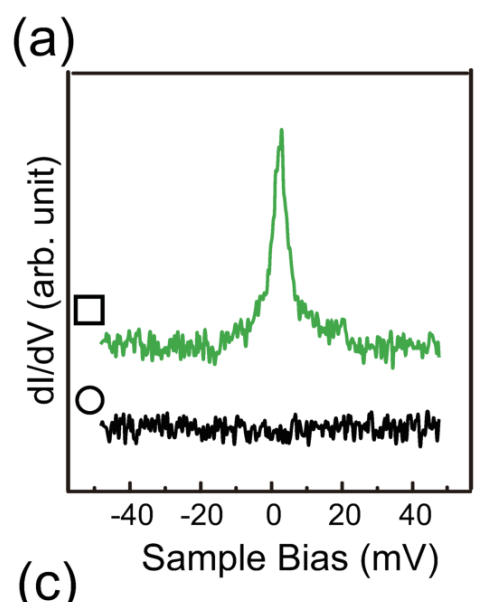

(b)

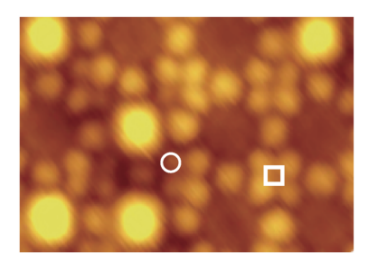

(d)
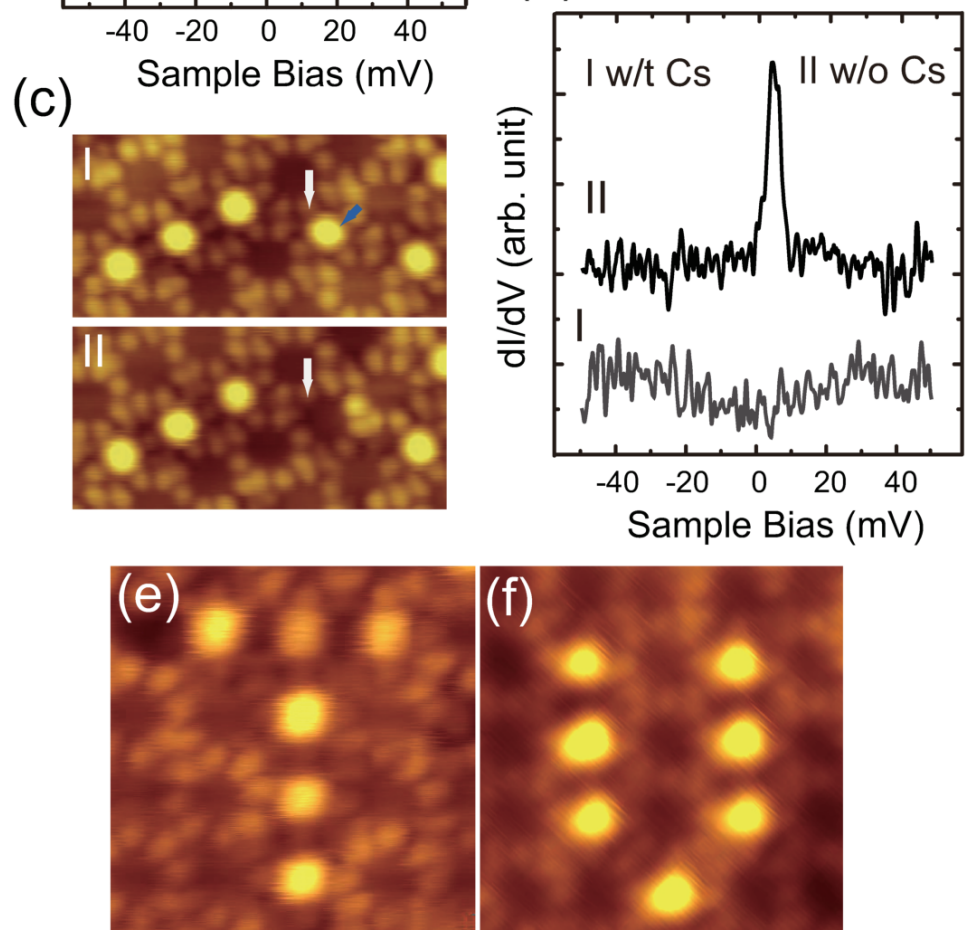

Figure 2: (a) STS spectra acquired over the locations shown in $(b)$. The square corresponds to a location close to the $\pi$-orbital of a $\theta=45^{\circ}$ molecule, where a zero-bias anomaly corresponding to a Kondo peak was observed, and the circle corresponds to a $\pi$-orbital of a $\theta=45^{\circ}$ molecule with an adsorbed Cs atom. No Kondo peak was observed. As in Ref. 7, $\theta=30^{\circ}$ molecules do not present Kondo peaks. (c) Before (I) and after (II) removal of a Cs atom from a molecule with $\theta=45^{\circ}$ using an STM tip. (d) Recovery of the Kondo peak after the removal of the Cs atom shown in $(c) .(e)$ and $(f)$ Initials of Tohoku University prepared by manipulating $\mathrm{Cs}$ atoms with the STM tip. 
is more compact.

Absorbed Cs atoms appeared as a protrusion in the center of the upper Pc ligand, as shown in Figure $1(f)$. From our total energy calculations, the Cs atoms are $0.54 \mathrm{eV}$ more stable on top of $\mathrm{YPc}_{2}$ than in direct contact with the metal substrate. In other words, it is more favorable that Cs binds directly to the top of the molecule, which is consistently observed in the STM images. There is a small preference of $0.12 \mathrm{eV}$ for the $\mathrm{YPc}_{2}$ molecules with $\theta=30^{\circ}$, which is not large enough to show a difference in the amounts of Cs atoms adsorbed on $\theta=30^{\circ}$ and $45^{\circ}$ molecules. Figure $1(f)$ shows three Cs atoms sitting on three consecutive $\mathrm{YPc}_{2}$ molecules with $\theta=30^{\circ}, \theta=45^{\circ}$, and $\theta=30^{\circ}$. Figures $1(g)$ and $(h)$ show simulated STM images with a $\mathrm{Cs}$ atom on every $\mathrm{YPc}_{2}$ molecule with $\theta=45^{\circ}$ and on those with $\theta=30^{\circ}$, respectively. The Cs atom lights up the molecule on which it sits. Thus, the molecules with $\theta=30^{\circ}$ develop a sharp contrast when Cs atoms are on top of them, and overlap with the image of the $\theta=45^{\circ}$ molecules causes a cross-like feature. This feature was clearly seen in the experimental STM images, as shown in Figure $1(i)$.

The molecules became brighter when a Cs atom was adsorbed because alkali atoms, like Cs, donate an electron to the molecules, increasing the density of states (DOS) of the occupied states. Evidence for charge transfer can be obtained from STS on the molecular layer, as shown in Figure 2, because the tunneling conductance above a given molecule is measured. In Figure $2(a)$, an STS spectrum of a molecule without a Cs atom (marked by a square) is plotted together with that of a molecule with one in a small bias window of $80 \mathrm{mV}$. Figure $2(b)$ shows the two locations at which STS were taken. In the STS spectrum, a zerobias anomaly due to a Kondo effect ${ }^{7}$ was observed, whereas no Kondo peak was observed for the doped molecule. In other words, a spin from the Cs atom is transferred to the SOMO of the $\mathrm{YPc}_{2}$ molecule, quenching the molecular magnetic moment. This interpretation is corroborated by the results of the DFT calculations. Bader analysis ${ }^{25}$ showed that there was an excess of 0.91 electrons on the doped $\mathrm{YPc}_{2}$ molecules.

We could manipulate the Cs at the atomic level with the STM tip by applying a pulse 
voltage (2.8 V, $10 \mathrm{~ms}$ duration, feedback-loop open) to the sample bias. Figure $2(c)$ shows an STM image of the controlled desorption of a Cs atom, which is marked with a blue arrow, and Figure $2(d)$ shows STS spectra taken before (marked with I) and after (marked with II) removal of the Cs atom. The Kondo peak reappeared when the Cs atom was removed from a $\mathrm{YPc}_{2}$ molecule with $\theta=45^{\circ}$. We further demonstrated that the STM tip could be used to manipulate dopants at the atomic level by arranging Cs atoms into the initials of Tohoku University, as shown in Figures 2 (d) and (e).

Figure 3 shows the doping behavior of the Cs atoms in relation to the DOS for the two molecules on the Au (111) surface and a comparison of the DOS and the STS spectra of the doped and undoped molecules. The upper panel in Figure $3(a)$ shows the DOS of a Cs-doped $\mathrm{YPc}_{2}$ molecule with $\theta=45^{\circ}$ and $\theta=30^{\circ}$. We have reported that the SOMOLUMO gap of a $\mathrm{YPc}_{2}$ molecule with $\theta=45^{\circ}$ is larger than that with $\theta=30^{\circ} .{ }^{7}$ Basically, the HOMO, SOMO, and LUMO of the two molecules are located between $-1 \mathrm{eV}$ and 1 $\mathrm{eV}$. When a Cs atom is adsorbed on a molecule with $\theta=45^{\circ}$, the SOMO becomes totally occupied and shifts $0.07 \mathrm{eV}$, and the HOMO shifts $0.17 \mathrm{eV}$ towards lower energies. These shifts do not affect the structure of the doped monolayer in our calculations, showing that the role of the Cs atom is to transfer one spin to the molecule.

Figures $3(b)$ and $(c)$ show the location at which STS were acquired and STS spectra, respectively. STS acquired over a molecule without a Cs atom are similar to those of the clean monolayer, showing that no long-range effect of the Cs atoms is felt by the molecules. On the other hand, when STS was performed over a doped molecule, the peak at ca. -0.5 $\mathrm{V}$ shifted by $\sim 0.2 \mathrm{~V}$ (marked with an open triangle), which is consistent with the calculated shift in the HOMO. In addition, the intensity of the LUMO peak dramatically increased (marked by an open circle). This is due to the dipolar interactions between the Cs ions and the molecules. Thus, the tunneling barrier for nearby electrons decreases, and the tunneling conductance increases for the higher energy orbitals. This effect is similar to the decrease in the work function of Cs-covered surfaces. 


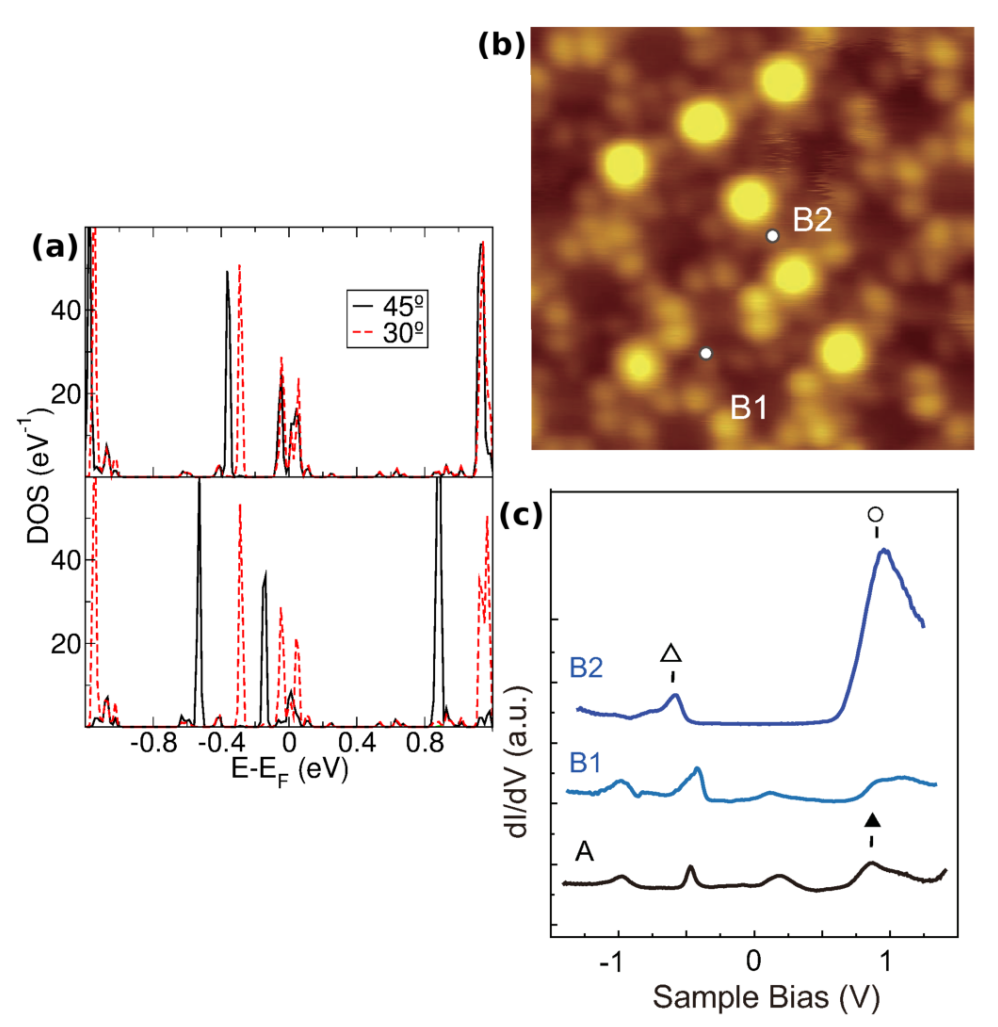

Figure 3: Electronic structure of the $\mathrm{YPc}_{2}$ monolayer with and without Cs atoms. (a) The upper panel shows the DOS of molecules with $\theta=45^{\circ}$ (black) and $\theta=30^{\circ}$ (red) without Cs atoms, and the lower panel shows the changes in the DOS upon adsorption of the Cs atoms on the $\theta=45^{\circ}$ molecule. The electronic structure close to the Fermi energy due to the molecular SOMO of the $\theta=45^{\circ}$ molecule shifts and becomes completely occupied. The HOMO and LUMO also shift, whereas the electronic structure of the molecule with $\theta=30^{\circ}$ does not change. (b) Locations on the surface where the STS spectra were acquired. $(c)$ STS spectra taken without Cs atoms (B1) are similar to those acquired at far away (A), meaning that the adsorbed Cs atoms do not have an effect on the monolayer. However, near the Cs atoms (B2) there are important effects: $(i)$ the electronic features at $-0.5 \mathrm{~V}$ shifted by $\sim 0.2 \mathrm{~V}$ towards lower energies, and (ii) the intensity of the peak at $1 \mathrm{~V}$ increased. The shift agrees with the theoretical one in $(a)$, and the increase in the peak height is due to a decrease in the tunneling barrier upon adsorption of a Cs atom. 
In summary, our combined experimental and theoretical work shows that Cs atoms prefer to adsorb on $\mathrm{YPc}_{2}$ molecules. Upon adsorption, the HOMO and SOMO levels shift due to charge transfer from the Cs atom. In other words, a spin is injected, quenching the intrinsic spin of the $\mathrm{YPc}_{2}$ molecules with $\theta=45^{\circ}$, which leads to disappearance of the Kondo peak of the tunneling conductance. Otherwise, the system remains largely unaffected except for a measurable increase in the conductance at higher biases due to a decrease in the tunneling barrier by the electric field of the Cs ion. Despite the large ratio of dopants to molecules, Cs atoms act as a spin dopant that changes the magnetic state of the $\mathrm{YPc}_{2}$ molecules with $\theta=45^{\circ}$.

\section{Acknowledgement}

T.K. and N.L. acknowledge the support from KAKENHI (22241026) and 'R\&D promotion scheme funding International joint research' promoted by NICT (National Institute of Information and Communication Technology), Japan. N.L. acknowledges support from the Spanish MICINN FIS2009-12721-C04-01. R.R. has been supported by a JAE-Doc contract

from the Consejo Superior the Investigaciones Científicas. R.R. and N.L. are supported by the ICT-FET Integrated Project AtMol (http://www.atmol.eu).

\section{References}

(1) Rocha, A. R.; García-Suárez, V. M.; Bailey, S. W.; Lambert, C. J.; Ferrer, J.; Sanvito, S. Nat Mater 2005, 4, 335-339.

(2) Wolf, S. A.; Awschalom, D. D.; Buhrman, R. A.; Daughton, J. M.; Von Molnár, S.; Roukes, M. L.; Chtchelkanova, A. Y.; Treger, D. M. Science 2001, 294, 1488-1495.

(3) Bogani, L.; Wernsdorfer, W. Nature Materials 2008, 7, 179-186. 
(4) Zyazin, A. S.; van den Berg, J. W. G.; Osorio, E. A.; van der Zant, H. S. J.; Konstantinidis, N. P.; Leijnse, M.; Wegewijs, M. R.; May, F.; Hofstetter, W.; Danieli, C.; Cornia, A. Nano Lett. 2010, 10, 3307-3311.

(5) Parks, J. J.; Champagne, A. R.; Costi, T. A.; Shum, W. W.; Pasupathy, A. N.; Neuscamman, E.; Flores-Torres, S.; Cornaglia, P. S.; Aligia, A. A.; Balseiro, C. A.; Chan, G. K. L.; Abruña, H. D.; Ralph, D. C. Science 2010, 328, 1370-1373.

(6) Comstock, M. J.; Strubbe, D. A.; Berbil-Bautista, L.; Levy, N.; Cho, J.; Poulsen, D.; Fréchet, J. M. J.; Louie, S. G.; Crommie, M. F. Physical Review Letters 2010, 104, 178301.

(7) Komeda, T.; Isshiki, H.; Liu, J.; Zhang, Y.; Lorente, N.; Katoh, K.; Breedlove, B. K.; Yamashita, M. Nat Commun 2011, 2, 217.

(8) Kawabe, M.; Masuda, K.; Namba, S. Japanese Journal of Applied Physics 1971, 10, $527-528$.

(9) Tang, C. W.; VanSlyke, S. A.; Chen, C. H. Journal of Applied Physics 1989, 65, $3610-3616$.

(10) Nollau, A.; Pfeiffer, M.; Fritz, T.; Leo, K. Journal of Applied Physics 2000, 87, 43404343.

(11) Gao, W.; Kahn, A. Applied Physics Letters 2001, 79, 4040-4042.

(12) Xue, J.; Forrest, S. R. Physical Review B 2004, 69, 245322.

(13) Murr, J.; Ziegler, C. Physical Review B 1998, 57, 7299-7304.

(14) Parthasarathy, G.; Shen, C.; Kahn, A.; Forrest, S. R. Journal of Applied Physics 2001, 89, 4986-4992. 
(15) Yan, L.; Watkins, N. J.; Zorba, S.; Gao, Y.; Tang, C. W. Applied Physics Letters 2001, $79,4148-4150$.

(16) Wüsten, J.; Berger, S.; Heimer, K.; Lach, S.; Ziegler, C. Journal of Applied Physics 2005, 98, 013705.

(17) Ding, H.; Gao, Y. Applied Physics Letters 2008, 92, 053309.

(18) Vitali, L.; Fabris, S.; Conte, A. M.; Brink, S.; Ruben, M.; Baroni, S.; Kern, K. Nano Letters 2008, 8, 3364-3368.

(19) Katoh, K.; Yoshida, Y.; Yamashita, M.; Miyasaka, H.; Breedlove, B. K.; Kajiwara, T.; Takaishi, S.; Ishikawa, N.; Isshiki, H.; Zhang, Y. F.; Komeda, T.; Yamagishi, M.; Takeya, J. J. Am. Chem. Soc. 2009, 131, 9967-9976.

(20) Kresse, G.; Furthmüller, J. Computational Materials Science 1996, 6, 15-50.

(21) Perdew, J. P.; Burke, K.; Ernzerhof, M. Physical Review Letters 1996, 77, 3865-3868.

(22) Grimme, S. Journal of Computational Chemistry 2006, 27, 1787-1799.

(23) Amft, M.; Lebègue, S.; Eriksson, O.; Skorodumova, N. V. Journal of Physics: Condensed Matter 2011, 23, 395001.

(24) (a) Tersoff, J.; Hamann, D. R. Physical Review Letters 1983, 50, 1998-2001; (b) Tersoff, J.; Hamann, D. R. Physical Review B 1985, 31, 805-813; (c) Bocquet, M.-L.; Lesnard, H.; Monturet, S.; Lorente, N. Computational Methods in Catalysis and Materials Science; Wiley-Vch: Weinheim, Germany, 2009.

(25) Bader, R. F. W. Atoms in Molecules: a Quantum Theory; Oxford University Press: New York, 1990. 